\title{
A Multi-agent System that Searches for Learning Objects in Heterogeneous Repositories
}

\author{
Fernando De la Prieta and Ana Belén Gil
}

\begin{abstract}
This paper presents the BRENHET application, which introduces a new concept in searching for educational resources by using a learning object paradigm that describes these resources. The application is composed of a complete agentbased architecture that implements the concept of federated search. It can search different repositories in parallel, and is based on abstraction layers between the repositories and the search clients.
\end{abstract}

Keywords: Multi-agent systems, Distributed Computing, e-learning, learning objects, repositories, Simple Query Interface, learning technology standards, web services.

\section{Introduction}

One of the biggest advances in the field of distance education has been the incorporation of the learning object paradigm[1]. The main advantage derived from the use of these elements is the reusability of the educational resources. These learning objects (LOs) incorporate both a self-contained modular resource and a set of descriptive metadata, which makes it possible to automate any search and sort process.

LOs are typically stored in digital repositories that, being highly heterogeneous, have different storing systems, access to objects, query methods, etc. The problem of heterogeneity in database systems has been the object of much research for quite some time [2] now and, as such, is not particularly critical. However, the fact that the repositories do not contain a high level layer that provides an abstraction between its internal functioning (which should be a black box) and the end users working with the stored data, results in a seemingly insurmountable problem suffered by the majority of repositories. In an attempt to avoid this problem, some

Fernando De la Prieta · Ana Belén Gil

Departamento Informática y Automática

Universidad de Salamanca

Plaza de la Merced s/n, 37008, Salamanca, Spain

e-mail: $\{$ fer, abg $\}$ @usal.es

Y. Demazeau et al. (Eds.): Trends in PAAMS, AISC 71, pp. 355-362. 
repositories have an abstraction layer between their internal and external characteristics, permitting them to better automate and process their learning objects, and allowing for new types of applications referred to as federated search LOs[3].

There are currently various studies underway that look at different ways of encapsulating heterogeneous learning object repositories (LORs). One of the most important, and currently the most used, is the SQI (Simple Query Interface) [4] which provides the repositories with an abstraction level between the internal and external heterogeneity that includes a specification with a wide range of possibilities (different service-based communication protocols, synchronous and asynchronous queries, stateless and stateful repositories, etc.).

After an extensive preliminary study, it is apparent that all current repositories use the VSQL[5] query language, which is both simple and powerful, since it is based on XML, and able to adapt perfectly to learning object searches. The semantic specification when searching for learning objects is the LOM (Learning Object Metadata) standard, which was formally approved by IEEE in 2002. Although LOM describes the LOs amply and correctly, it does not contain a required specification field. Thus the burden of correctly describing the educational resources is placed squarely on its authors.

This primary purpose of this article is to present the federated search system for LOs in digital repositories, which has been developed specific to this study. Known as BRENHET, the agent-based system uses the SQI interface to perform federated searches in different repositories and sort the retrieved LOs according to the different needs of the application users.

\section{Searching for Learning Objects in Repositories}

The current increase in resources for online education calls for the efficient management of those services and the elements involved. The greatest efforts within the context of e-learning are now focused on achieving the interoperability of the resources generated. It is within this context that LOs emerged with the aim of reusing educational resources and reducing their production costs [7].

LOs have to be labeled with metadata so that they can be identified, located and used for educational purposes in web-based contexts. The pioneering standardization for LOs was developed by ARIADNE (Alliance of Remote Instructional Authoring and Distribution Networks for Europe). The Instructional Management Systems project (IMS) and Advanced Distributed Learning (ADL) initiatives emerged in an effort to develop standards for distributed learning technologies. The former has become the IMS Global Learning Consortium, whose key work has been in vocabularies and metadata for learning objects (IMS Learning Resource Metadata specification). The latter is the home of the SCORM (Sharable Content Object Reference Model), a Web-oriented data model for content aggregation focusing on the structure and run-time environment for LOs. SCORM is pretty much accepted as the standard for management of educational content, and its counterpart is a standard for management of educational content metadata [8], Learning Object Metadata (LOM) from IEEE Learning Technologies Standards Committee (LTSC). 


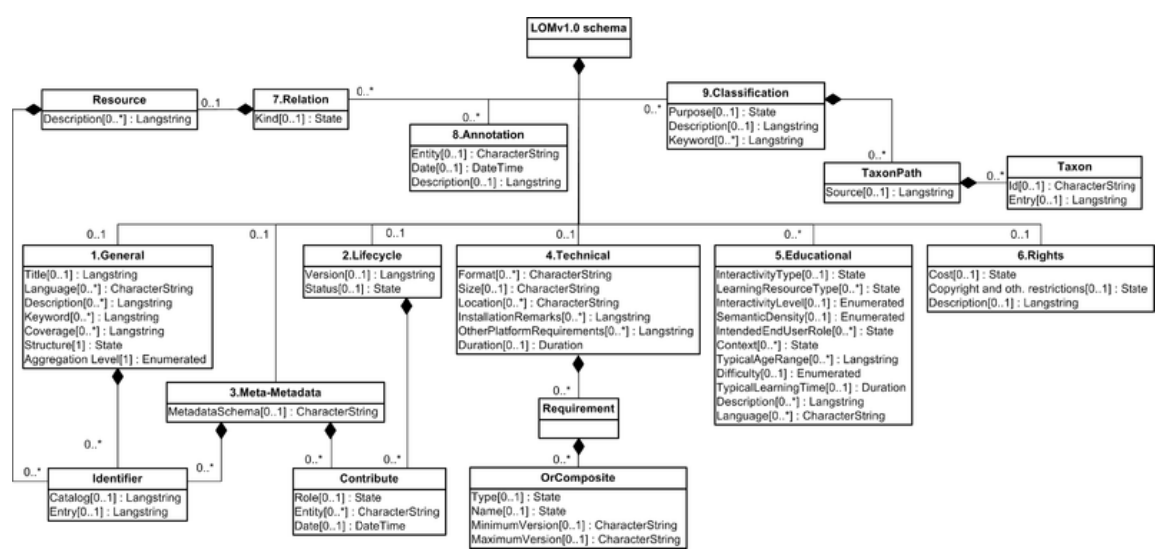

Fig. 1 LOM (Learning Object Metadata)

Both LOM and SCORM were written to be very flexible and extensible, so although learning objects have many variant recommendations, they also have a remarkably large shared foundation.The IEEE LOM standard (IEEE Draft 1484.12.1, 2002), specifies the conceptual schema that defines the structures of the data for instances of LO metadata. The basic schema of LOM is composed of categories and elements as shown in Figure 1, which can describe the resources very well. LOM is able to embed other metadata standards using XML namespaces.

\subsection{Learning Objects Repositories}

LORs are software systems in the form of digital catalogues that either store educational resources and their metadata, or only the latter. Most LOR belong to educational institutions, as is the case of MERLOT (Multimedia Educational Resource for Learning and Online Teaching), CAREO (Campus Alberta Repository of Education Objects), or CLOE (Co-operative Learning Object Exchange). They generally provide some kind of search interface that makes it possible to retrieve the LOs. Any interaction for retrieving LOs can be done manually or be automated through different software systems. The main standards of interfaces for LORs are CORDRA, IMS-DRI, OKI-OSID or SQI.

- CORDRA (Content Object Repository Discovery and Registration/Resolution Architecture). This develops an open model for the design and implementation of software systems that retrieve, share and reutilise educational contents through the implementation of federated systems in repositories of educational resources.

- IMS-DRI (IMS Digital Repositories Specification). IMS Digital Repositories v1.0 are aimed at providing recommendations for the interoperability of the most common repository functions. These recommendations should be implemented through web services so that they have a common interface. On the 
broadest level, this specification defines digital repositories as being any collection of resources that are accessible via a network without prior knowledge of the structure of the collection.

- OKI OSID (Open Knowledge Initiative Open Service Interface Definition). The OKI specification allows sustainable interoperability and integration by defining standards based on Service Oriented Architecture (SOA). OSID is simply the contract software and thus is compatible with most other technologies and specifications, such as SOAP and WSDL.

- SQI (Simple Query Interface), defined in November 2005 by CEN (European Committee of Standardization), is defined by three APIs: Learning Objects Interoperability Framework, Authenticationand Session Management, and Simple Query Interface Specification, which provides a detailed description of the functionalities that each repository must meet in order to be compatible with the others, and describes the names of the methods that must be implemented along with the parameters received and returned during the search process.

It is thus imperative to be able to rely on an efficient and interoperable search system that can guarantee the recovery of learning objects from the repositories. For this reason we have designed an SQI-based metasearch system that can perform a federated search in distributed repositories.

\section{Overview of BRENHET}

We have built a LO federated search system for digital repositories that is completely functional and accessible by any user. In addition to searching for LOs, the system catalogues objects that are returned by the LORs, thus facilitating the final selection made by the end users. The final sorting is based on two variables. The first estimates the quality of the objects according to different parameters such as size, included key characteristics, etc. The second variable looks at the end user activity to classify the recovered objects. This provides the classification process with techniques that come directly from a collaborative recommendation of network information (Collaborative-Social-filtering systems[6]).

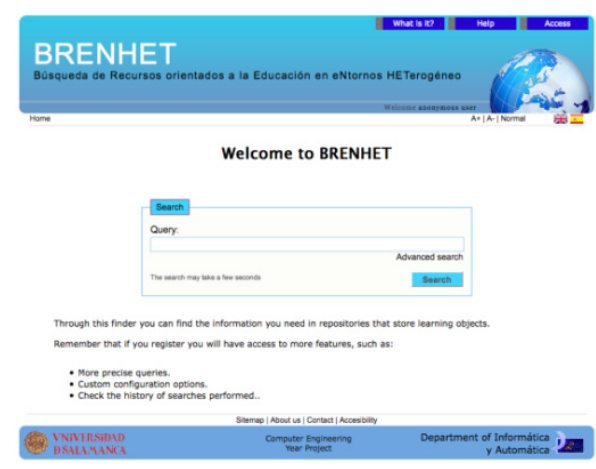

Fig. 2 BRENHET. Federated search system. 
Two search interfaces are provided. One is simple and quick, allowing the user to input a series of key words or an exact phrase that should contain the metadata of both. There is also a more advanced and powerful search that provides the user with a greater number of search options.

As part of its administrative functionality, the BRENHET system implements a new repository management system that provides the application with an inclusion protocol for new repositories. This protocol incorporates an automated validation system of repositories prior to their inclusion, that ensures the application and the search system are autonomous and reliable.

The functionality of the application is enhanced and its use greatly facilitated by the implementation of the following additional functionalities: user management, configuration management, search history, and a complete system of statistics based on statistical data gathered in each query from each repository.

\subsection{BRENHET Search Architecture}

In order to develop the BRENHET application, it was necessary to design and implement an agent-based architecture specifically focused on resolving the problem of the federated search in repositories that store learning object through the use of a SQI query interface.

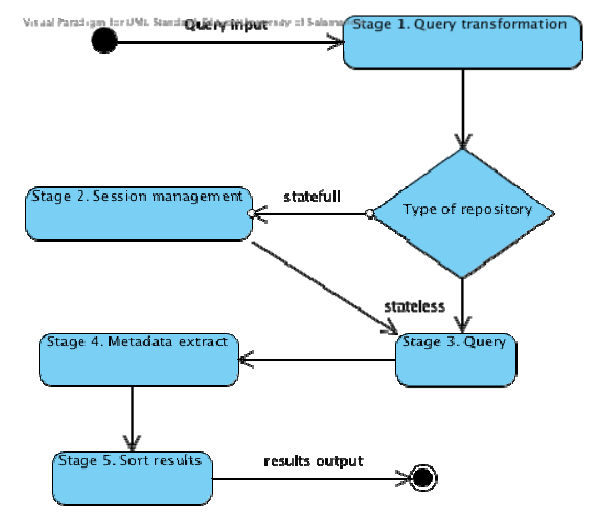

Fig. 3 Sequence of task in federated search

As shown in the figure above, the query and the results output processes are similar for each query and include the following phases:

1. Query transformation. During the development of this phase, the query is transformed into a more common language that the user inputs in the application. It is a VSQL language that, as previously mentioned, is the query language for repositories that implement the SQI interface.

2. Session management. This phase only needs to be executed in statefull repositories. The identifier is obtained through a query using web services. 
3. Query. Once the session identifier, whether static or dynamic, is available, the next step that must becarried out is the actual query. A synchronous query is implemented so that the results are obtained as an answer to the web services query.

4. Metadata extract. Once the results have been obtained, the information contained in the metadata for each learning object is extracted.

5. Sortresults. Once all the learning objects are obtained, the results are ordered so that they can be presented to the user more properly. This selection process must consider the use made of the learning objects by the end users, and the quality of the objects themselves.

Phase 1 transforms natural language into VSQL language. Within the context of this type of query, the transformation phase is performed in a direct manner, which can be fully appreciated in Figure 4.

$$
\begin{aligned}
& \text { dog "learnin- } \\
& \text { gobject" } \\
& \begin{array}{l}
\rightarrow \quad \text { "dog" or "lear- } \\
\text { ningobject" }
\end{array} \\
& \begin{aligned}
& <\text { simpleQuery }> \\
& <\text { term }>\text { dog }<\text { /term }> \\
& <\text { term }>\text { learningobject }</ \text { term }> \\
& <\text { /simpleQuery }>
\end{aligned}
\end{aligned}
$$

Fig. 4 VSQL transformation

Phases 2 and 3 of the algorithm constitute the majority of the time used in the query, since they make a series of requests to the LORs using web services. Phase 4 processes the results so that they can be more coherently ordered in the subsequent phase. This is possibly the most important phase of the algorithm since it considers various premises that can be divided into two primary groups:

1. Importance for the user. As users navigate the application, data is gathered from the use of LOs and later used during the ordering phase.

2. Quality of the LOs. The quality of the actual learning object is considered (key elements, number of elements, relationship with other objects, etc.).

During the algorithm design phase, the response time of the repositories was too high, just as with the results of initial study. One solution to this problem was to deploy the previously described phases 2,3 and 4 in parallel, and to implement a timeout system. This reduced the response time to the end user and resulted in a completely functional application.

The BRENHET search system architecture is composed of three basic blocks: Interface, Search system, and Communication protocols. These blocks constitute the foundation of the system's functionality. The interface is primarily used for a bidirectional communication between the users and the application. The search system is implemented through the use of agents that are responsible for security, communication and the taskflow in BRENHET. This block is the core of the application and integrates two types of agents that are each assigned to different types of tasks according to each one's primary objective. The first group is 
composed of deliberative BDI agents, which are responsible for keeping track of and coordinating the areas, can modify their behavior according to the knowledge acquired in order to find the best solution. These agents base their decision on the knowledge acquired and on a series of statistical data that is gathered during each interaction with the repositories that contain LOs. This provides the architecture with great flexibility, even for incorporating new agents in the future, which helps improve the overall time and functioning of the global search process.

The following list describes the predefined agents that provide the basic functionality of the BRENHET architecture:

- Comunication Agent. Responsible for all the communication between the different repositories and the application. Manages all of the requests and responses simultaneously. These agentsuse Web services to communicate, and send and receive messages in XML format.

- Security Agent. Responsible for verifying the XML message sreceived and confirming that they are correct, and that the LOs are of a good enough quality to be reused.

- Statistical Agent. Responsible for taking statistical data from each exchange of communication with the repositories, and presenting the data to the other agents so that they can make the best decisions at any given time.

- Sort Agent. Responsible for sorting the results according to the statistical data on the use of the learning objects made by the end users.

- Supervisor Agent. This agent's task is to supervise and ensure that the other agents are functioning correctly and that the application provides the desired functionality.

- Optimize Agent. Responsible for optimizing system performance with respository queries.
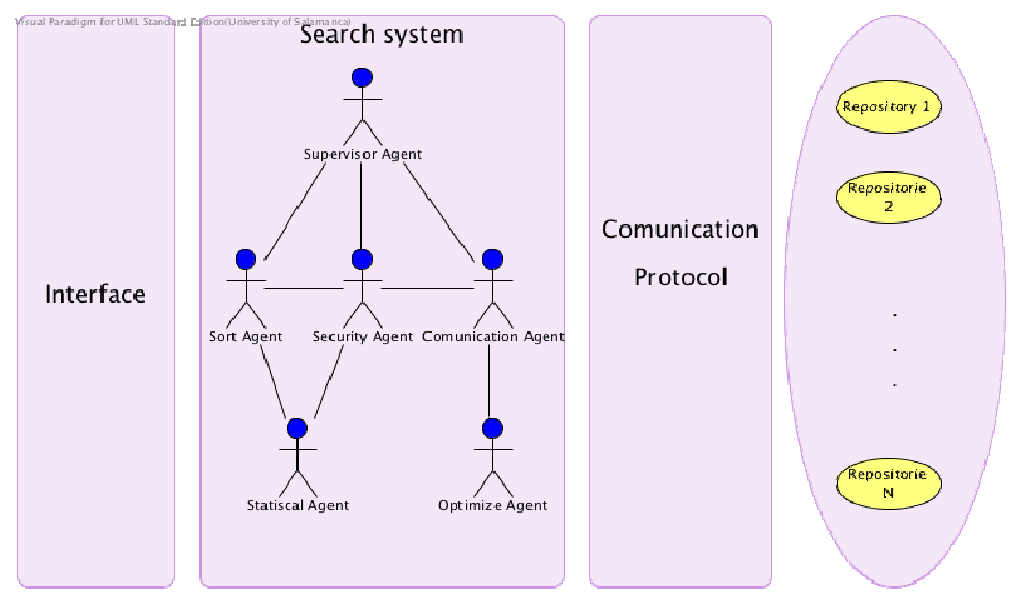

Fig. 5 BRENHET Architecture 
The second group of agents is based on a reactive agent platform that primarily aims to improve the application's overall response time. These agents must take into account a series of predefined temporal restrictions so that they can be capable of autonomously controlling the overall quality and reliability of the application.

Finally, the communication protocol permits the applications, services and sensors to be continually interconnected with the agent-based search system. This protocol is based on the SOAP standard and allows the exchange of messages between applications, services and repositories.

\section{Results and Conclusions}

This paper has provided a brief glimpse into the BRENHET System, which resolves the problem of heterogeneity in repositories that contain LOs, and the difficulty in processing the repositories that arises from the inconsistencies within the LOM specification. BRENHET is a system that searches for LOs with a federated search. It has an agent-based architecture that uses an SQI interface to access Web services for heterogeneous repositories that store LOs, to select the required objects, and sort them according to user preferences.

Taking into account the results obtained from the construction of the BRENHET application, it is possible to conclude that the agent-based architecture is ideal for resolving the problem of federated searches in heterogeneous repositories, due to the adaptation and learning facilities of the actual agents.

\section{References}

1. Lujara, S.K., Kissaka, M.M., Bhalaluseca, E.P., Trojer, L.: Learning Objects: A new paradigm for e-learning resource development for secondary schools in Tanzania. World Academy or Sciencie, Engineering and Technology, 102-106 (2007)

2. Abelló, A., Oliva, M., Samos, J., Saltor, F.: Information System Architecture for Data Warehousing from a Federation. In: Int. Workshop on Engineering Federated Information Systems, EFIS 2000 (2000)

3. Gil-González, A.-B., García-Peñalvo, F.: Multiagent system for learning objects recovery with context attributes. Int. J. Computer Applications in Technology 33(4), 320-326 (2008)

4. Campi, A., Ceri, S., Dolog, P., Duval, E., Guinea, S., Houben, G., Massart, D., Nilsson, M., Ternier, S., Xuan, Z.: ARIADNE foundation for the European Knowledge Pool. ProLearning Query Language Definition, V.0.7, http: / /

ariadne.cs.kuleuven.be/lomi/index.php/QueryLanguages_stable

5. Simon, B., Massart, D., Van Assche, F., Ternier, S., Duval, E., Brantner, S., Olmedilla, D., Miklos, Z.: A Simple Query Interface for Interoperable Learning Repositories. In: 1st Workshop on Interoperability of Web-based Educational Systems, Chiba. Japan (2005)

6. Herlocker, J.L., Konstan, J.A., Terveen, L.G., Riedl, J.T.: Evaluating collaborative filtering recommender systems. ACM Trans. Inf. Syst. 22(1), 5-53 (2004)

7. Hatala, M., Richards, G., Eap, T., Willms, J.: The interoperability of learning object repositories and services: standards, implementations and lessons learned. In: Proceedings of the 13th international World Wide Web Conference on Alternate Track Papers \&Amp; Posters, WWW Alt. 2004, May 19 - 21, pp. 19-27. ACM, New York (2004)

8. Ogbuji, U.: Think XML: Learning Object metadata, XML Knowledge management flourishes in learning technology initiatives (2003), http://www.ibm.com/ developerworks / xml/library/x-think21.html 